\title{
Irreversible Respiratory Failure in a Full-Term Infant with Features of Pulmonary Interstitial Glycogenosis as Well as Bronchopulmonary Dysplasia
}

\author{
Maresa E. C. Jiskoot-Ermers, MD ${ }^{1}$ Tim A. J. Antonius, MD ${ }^{1}$ Monika G. Looijen-Salamon, MD² \\ Marc H. W. A. Wijnen, MD, PhD ${ }^{3}$ Bettina F. Loza, MD ${ }^{4}$ Arno F. J. van Heijst, MD, PhD ${ }^{1}$
}

${ }^{1}$ Department of Pediatrics, Radboud University Medical Centre,

Address for correspondence Maresa E. C. Jiskoot-Ermers, MD,

Nijmegen, The Netherlands

2 Department of Pathology, Radboud University Medical Centre, Department of Pediatrics, Radboud University Medical Centre, Post Box 9101, 6500 HB, Nijmegen, The Netherlands

Nijmegen, The Netherlands

${ }^{3}$ Department of Pediatric Surgery, Radboud University Medical (e-mail: Maresa.jiskoot@radboudumc.nl).

Centre, Nijmegen, The Netherlands

${ }^{4}$ Department of Pediatrics, VieCuri Medical Centre, Venlo,

The Netherlands

Am J Perinatol Rep 2015;5:e136-e140.

\begin{abstract}
Pulmonary interstitial glycogenosis (PIG) is a rare interstitial lung disease in the newborns. We report on the clinical presentation and pathological findings of a full-term male infant with pulmonary hypertension requiring extracorporeal membrane oxygenation (ECMO). An open lung biopsy demonstrated interstitial changes resembling pulmonary interstitial glycogenosis as well as bronchopulmonary dysplasia (BPD), without convincing evidence of maturational arrest, infection, alveolar proteinosis, or alveolar capillary dysplasia. The boy

Keywords

- pulmonary interstitial glycogenosis

- respiratory failure

- interstitial lung disease

- ECMO

- pulmonary hypertension was treated with glucocorticoids and, after a few days, was weaned from ECMO. A few hours later, the patient died due to acute severe pulmonary hypertension with acute right ventricular failure. The etiology and underlying pathogenic mechanisms of PIG are unknown. The clinical outcomes are quite varied. Deaths have been reported when PIG exists with abnormal lung development and pulmonary vascular growth and congenital heart disease. No mortality has been reported in PIG together with BPD in full-term infants. In this article, we reported on a full-term infant with interstitial changes resembling PIG and BPD who expired despite no convincing evidence of an anatomical maturational arrest or congenital heart disease.
\end{abstract}

Respiratory insufficiency in term infants is usually caused by meconium aspiration, sepsis, or pneumonia. Interstitial lung disease, a heterogeneous group of diffuse lung diseases, is a rare cause of respiratory insufficiency in newborns. A new classification of this group of lung diseases was established by the Children's Interstitial Lung Disease Network. ${ }^{1-6}$

In this case report, we present a full-term infant with pulmonary interstitial glycogenosis (PIG) together with bronchopulmonary dysplasia (BPD) like features.

received

July 9,2014

accepted after revision

March 19, 2015

published online

June 3, 2015
$10.1055 / \mathrm{s}-0035-1551674$ ISSN 2157-7005.

\section{Case Report}

An 11-day-old Moroccan boy was transferred from another neonatal intensive care unit (NICU) due to refractory pulmonary hypertension requiring extracorporeal membrane oxygenation (ECMO). It was the mother's fourth pregnancy, which was uneventful. The parents were first cousins, there is no family history of respiratory problems. The boy was born at a gestational age of 39 weeks and 3 days. Birth weight was

Copyright $\odot 2015$ by Thieme Medical Publishers, Inc., 333 Seventh Avenue, New York, NY 10001, USA. Tel: +1(212) 584-4662.
License terms

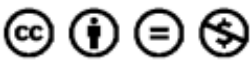


3,730 g and Apgar scores were 9 and 10 at 1 and 5 minutes, respectively. Initially, he was discharged home without problems. After 1 day, parents sought medical attention due to tachypnea and feeding problems, after which he was admitted to the NICU for hypoxia. Cardiac ultrasound showed normal cardiac anatomy and a patent ductus arteriosus (PDA) with signs of persistent pulmonary hypertension of the newborn (PPHN). Inhaled nitric oxide and inotropic support were started. Antibiotics were started empirically, though there were no signs of viral or bacterial infection. With these interventions, his condition improved, but after 2 days, he became hypoxic again due to severe pulmonary hypertension with right-to-left shunting through the PDA. Surfactant was administered without any beneficial effect. Despite intensive treatment, the PPHN could not be reversed, and the boy was referred to our center for extracorporeal membrane oxygenation (ECMO). Cardiac ultrasound showed a severe persistent pulmonary hypertension with suprasystemic pressures, enlarged right ventricle, and a continuous right-to-left shunting through the PDA. Veno-venous ECMO was started on day 10.

Chest radiographs (-Fig. 1) showed diffuse pulmonary opacities consistent with atelectasis or edema. Because it was impossible to wean the patient from ECMO support and due to lack of a definitive diagnosis, an open lung biopsy was performed on day 8 of ECMO treatment.

Histological examination of the biopsy showed partially atelectatic and partially emphysematous lung parenchyma, including interstitial emphysema. Radial alveolar count (RAC, i.e., the number of alveoli in a subpleural acinus between the central bronchiole and the visceral pleura) could be measured at only one place in the biopsy and was four to five, which is within the normal parameters (-Fig. 2a). Except for a few remnants of hyaline membranes, the alveoli were empty. Pneumocytes were enlarged and showed large round nuclei with prominent nucleoli. The alveolar septa were widened due to three factors: first, there were several accumulations of histiocytic cells with abundant, clear cytoplasm. Second, the amount of collagen fibers was increased as demonstrated by
Elastica-Masson stain (-Fig. 2b). Finally, there was an increase of interstitial smooth muscle bundles as shown by an immunohistochemical SM1-staining (-Fig. 2c). Small arterioles showed a constricted media, but no significant intimal proliferation or fibrosis. An immunohistochemical CD34 staining highlighted numerous capillaries at considerable distance from the alveolar surface, yet evenly distributed in the septa. There was no misalignment of venous vessels. A periodic acid Schiff (PAS) stain revealed some granular material digestable by diastase, consistent with glycogen, in the clear cytoplasm of the interstitial histiocytes ( - Fig. 2e, f) In summary, the presence of PAS-positive histiocytes led to the diagnosis of PIG, whereas the interstitial increase of collagen and smooth muscle bundles were interpreted as reparative phase of BPD. ${ }^{7}$ Based on this biopsy, there was no convincing evidence of an anatomical maturational arrest, infection, alveolar proteinosis, or alveolar capillary dysplasia. The distribution of the PAS positive histiocytes was somewhat irregular, the largest concentrations being located at the intersections of alveolar septa.

The boy was treated with glucocorticoids due to the reported potential beneficial effect in cases of PIG. ${ }^{2,8-12}$ After a few days of prednisolone, we were able to take the patient off ECMO. A few hours later, the patient again developed acute severe pulmonary hypertension with acute right ventricular failure. There was no evidence of a pulmonary embolism at this time. Maximal ventilator support with inhaled nitric oxide failed, and the patient expired. Autopsy was not permitted by the patient's family.

\section{Discussion}

Pulmonary interstitial glycogenosis (PIG) is a rare and poorly understood lung disorder. ${ }^{5,8,12,13}$ Diagnosis can only be made by means of a lung biopsy. Its characteristic histological finding is interstitial thickening due to increased mesenchymal cellularity without evidence of inflammatory cells. The inter-alveolar septa are expanded by oval- to spindle-shaped cells with pale cytoplasm, indistinct cell membranes, and

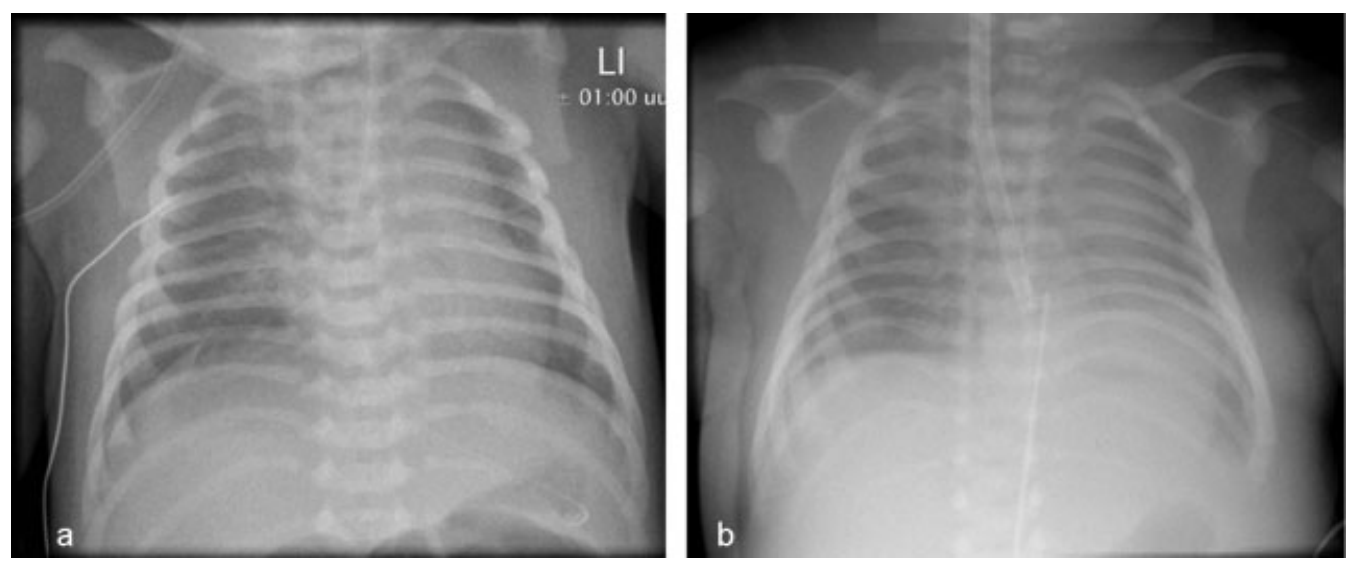

Fig. 1 Chest radiographs. (a) Portable frontal chest radiograph 1 day after birth showed a normal configuration of the heart. There are some prominent interstitial markings bilateral. (b) Portable frontal chest radiograph 10 days after birth. Endotracheal tube is in a high position. ECMO canula is in a good position. Interstitial markings bilateral consistent with atelectasis or edema. ECMO, extracorporeal membrane oxygenation. 

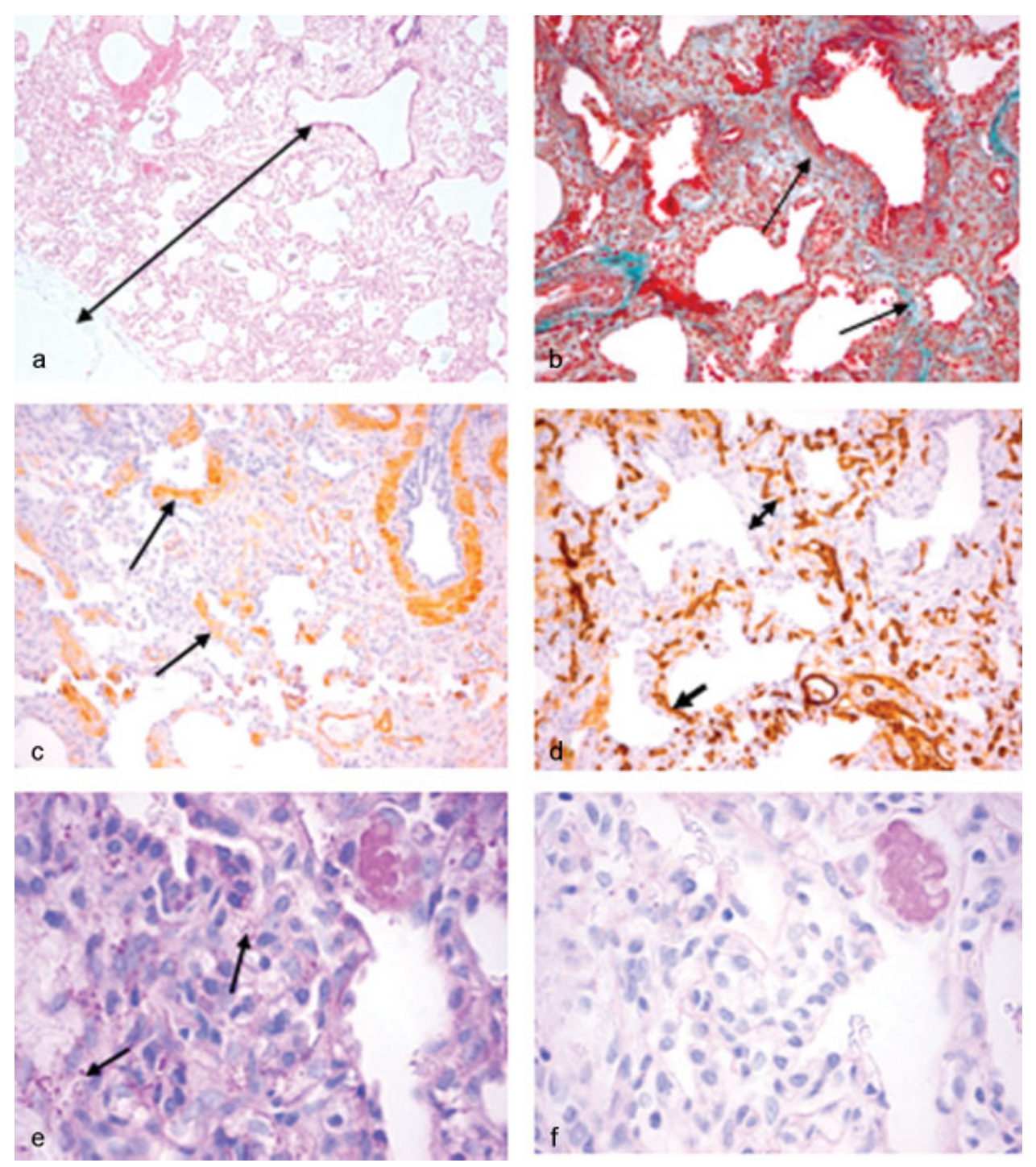

Fig. 2 Histological aspects of open lung biopsy. (a) ( $\times 50$, H\&E stain) scanning view: double arrow indicates radial alveolar count; (b) $(\times 100$, Elastica-van Masson stain): increase of interstitial collagen fibers (arrows); (c) immunohistochemistry: arrows indicate increase of interstitial smooth muscle cells ( $\times 100$, SM1 stain); (d) immunohistochemistry: some capillaries (simple arrow) are in touch with alveolar basal membrane, but many (double arrow) are not $(\times 100$, CD34 stain); (e and f) (PAS and PAS-D, $\times 200)$ interstitial cells with PAS-positive cytoplasmatic granules (arrows in - Fig. 2e) which disappear after diastase treatment.

oval, bland nuclei. Staining with PAS shows patchy PASpositive diastase sensitive material within the cytoplasm of these interstitial cells, indicating the presence of glycogen. The true function of these interstitial cells is unclear, but is probably similar to fibroblastic cells. ${ }^{1,3,5,8,9}$ The interstitial cells found in our patient's lung biopsy matched this description, although the amount of cytoplasmatic PAS digestable granules was not very large. We could not perform electron microscopy, because our standard fixation protocol for perinatal lung biopsies does not contain glutaraldehyde. There are two patterns of PIG observed: a diffuse interstitial PIG without growth abnormalities or patchy PIG involvement in preterm patients with alveolar growth abnormalities. ${ }^{4,14,15}$ In our small biopsy, it was difficult to determine the overall distribution pattern of the diagnostic cells; we can state that it was not entirely diffuse. PIG has been described as both an isolated finding and an additional finding in abnormal lung development and pulmonary vascular growth, hemodynamically significant cardiac disease, Hunter syndrome and Noonan syndrome. ${ }^{4,11-13,15-19}$ At histological investigation of our biopsy, the predominant features were interstitial changes of the lung parenchyma resembling BPD, which we don't expect in a full term infant. However, the boy was all the time artificially ventilated with high pressures potentially contributing to lung damage which made differentiation between a developmental disorder and secondary changes due to ventilation difficult. Therefore, as parameter of maturity, we used the RAC, which was within normal limits. We are aware of the fact that there were only two bronchioli visible in the slides; however, we assumed that we would have seen more bronchioles if there had been significant delay in the development of alveoli. The walls of the smallest arterioles 
were thickened due to an increase of smooth muscle cells. We considered these changes consistent with pulmonary hypertension as a consequence of the interstitial changes. There were no signs of alveolar capillary dysplasia and no misalignment of pulmonary veins. Unfortunately, since autopsy was not permitted, there was no further possibility to obtain more lung tissue to exclude a sampling error. In summary, our patient is a full-term infant with histological features of PIG and bronchopulmonary dysplasia without an anatomical maturation arrest or a misalignment of pulmonary veins. But because of the technical limitations mentioned above, and since no genetic tests for surfactant genes were performed we cannot rule out a functional immature lung nor can we decide with certainty if PIG alone caused PPHN or if PPHN was due to the BPD like changes and PIG has to be considered as an epiphenomenon. However, histological signs of surfactant deficiency were not seen and PPHN was already present shortly after birth, at a time point where eventual BPD was not yet present.

All patients presenting with PIG demonstrated tachypnea and hypoxemia, most of them immediately after birth or in the first 2 days of life. It has not been seen past 8 months of age. The majority required assisted ventilation. ${ }^{1,4,6,8,9}$ In the relatively small number of reported cases, there is a male gender preference. ${ }^{8,10,20}$

Radiological findings of PIG are nonspecific. They vary considerably, depending on whether there is any associated underlying growth abnormality. Infants with PIG not associated with an underlying growth abnormality show progressive bilateral hyperinflation and eventual evolution to diffuse interstitial markings on their chest X-rays. High-resolution computed tomography (CT) scan of the chest demonstrates segmental, or subsegmental ground-glass opacities, interlobular septal thickening, and reticular changes in a primarily subpleural distribution with few centrilobular nodules. Infants with PIG associated with underlying growth abnormalities, show multiple small scattered cystic changes of variable size combined with underlying diffuse ground-glass opacity, interlobular septal thickening and reticular changes on $\mathrm{CT}^{6,8,9,14}$

The etiology and underlying pathogenic mechanism of PIG are unknown. There have been no reported associations with glycogenoses or other metabolic diseases. The current hypothesis is that this lung abnormality originates in utero and is a developmental abnormality. ${ }^{4,8-13,16}$ Maybe is patchy PIG associated with anatomical abnormal lung development and pulmonary vascular growth is an early development disorder and diffuse isolated PIG a late development disorder.

Currently, there are no guidelines for the treatment of PIG. Administration of glucocorticoids has been recommended and has demonstrated a favorable outcome in affected patients. ${ }^{2,8-12}$

The prognosis remains uncertain. The clinical outcomes are quite varied. Deaths have been reported when PIG exists with an abnormal lung development and pulmonary vascular growth, and congenital heart disease. No mortality has been reported in isolated PIG. ${ }^{4,11,13,19}$
In this article, we reported on a full-term infant with features of PIG and BPD, who expired. There was no convincing evidence of an anatomical maturational arrest or a congenital heart disease. It is also possible that our patient had an undiagnosed genetic or pulmonary abnormality. So maybe PIG is an epiphenomenon at lung development disorders in different stages and is the prognosis depending on the stage of the development disorder. ${ }^{16,18,20}$

In conclusion, our knowledge of incidence, etiology, and pathogenesis of PIG is lacking. The diagnosis can be made only after lung biopsy demonstrates the characteristic histological findings. The prognosis of PIG continues to evolve. Further investigation beyond simple case reports is needed to thoroughly characterize this disorder.

Note

It is also possible that our patient had an undiagnosed genetic or pulmonary abnormality.

\section{References}

1 Deutsch GH, Young LR, Deterding RR, et al; Pathology Cooperative Group; ChILD Research Co-operative. Diffuse lung disease in young children: application of a novel classification scheme. Am J Respir Crit Care Med 2007;176(11):1120-1128

2 Das S, Langston C, Fan LL. Interstitial lung disease in children. Curr Opin Pediatr 2011;23(3):325-331

3 Dishop MK. Paediatric interstitial lung disease: classification and definitions. Paediatr Respir Rev 2011;12(4):230-237

4 Popler J, Lesnick B, Dishop MK, Deterding RR. New coding in the International Classification of Diseases, Ninth Revision, for children's interstitial lung disease. Chest 2012;142(3):774-780

5 Cazzato S, di Palmo E, Ragazzo V, Ghione S. Interstitial lung disease in children. Early Hum Dev 2013;89(Suppl 3):S39-S43

6 Lee EY. Interstitial lung disease in infants: new classification system, imaging technique, clinical presentation and imaging findings. Pediatr Radiol 2013;43(1):3-13, quiz 128-129

7 Travis WD, Colby TV, Koss MN, Rosado-de-Christenson ML, Müller NL, King TE. Non-Neoplastic Disorders of the Lower Respiratory Tract. Atlas of Nontumor Pathology. Vol 2. Washington DC: The American Registry of Pathology; 2002

8 Canakis AM, Cutz E, Manson D, O’Brodovich H. Pulmonary interstitial glycogenosis: a new variant of neonatal interstitial lung disease. Am J Respir Crit Care Med 2002;165(11):1557-1565

9 Smets K, Dhaene K, Schelstraete P, Meersschaut V, Vanhaesebrouck P. Neonatal pulmonary interstitial glycogen accumulation disorder. Eur J Pediatr 2004;163(7):408-409

10 Onland W, Molenaar JJ, Leguit RJ, et al. Pulmonary interstitial glycogenosis in identical twins. Pediatr Pulmonol 2005;40(4): 362-366

11 Radman MR, Goldhoff P, Jones KD, et al. Pulmonary interstitial glycogenosis: an unrecognized etiology of persistent pulmonary hypertension of the newborn in congenital heart disease? Pediatr Cardiol 2013;34(5):1254-1257

12 Citti A, Peca D, Petrini S, et al. Ultrastructural characterization of genetic diffuse lung diseases in infants and children: a cohort study and review. Ultrastruct Pathol 2013;37(5):356-365

13 King BA, Boyd JT, Kingma PS. Pulmonary maturational arrest and death in a patient with pulmonary interstitial glycogenosis. Pediatr Pulmonol 2011;46(11):1142-1145

14 Castillo M, Vade A, Lim-Dunham JE, Masuda E, Massarani-Wafai R. Pulmonary interstitial glycogenosis in the setting of lung growth 
e140 PIG is a Rare Interstitial Lung Disease in Newborns Jiskoot-Ermers et al.

abnormality: radiographic and pathologic correlation. Pediatr Radiol 2010;40(9):1562-1565

15 Ross MK, Ellis LS, Bird LM, Hagood JS. Pulmonary interstitial glycogenosis in a patient ultimately diagnosed with Noonan syndrome. Pediatr Pulmonol 2014;49(5):508-511

16 Deutsch GH, Young LR. Pulmonary interstitial glycogenosis: words of caution. Pediatr Radiol 2010;40(9):1471-1475

17 Smets K, Van Daele S. Neonatal pulmonary interstitial glycogenosis in a patient with Hunter syndrome. Eur J Pediatr 2011;170(8): 1083-1084
18 Alkhorayyef A, Ryerson L, Chan A, Phillipos E, Lacson A, Adatia I. Pulmonary interstitial glycogenosis associated with pulmonary hypertension and hypertrophic cardiomyopathy. Pediatr Cardiol 2013;34(2):462-466

19 Ehsan Z, Montgomery GS, Tiller C, Kisling J, Chang DV, Tepper RS. An infant with pulmonary interstitial glycogenosis: clinical improvement is associated with improvement in the pulmonary diffusion capacity. Pediatr Pulmonol 2014;49(3):E17-E20

20 Lanfranchi M, Allbery SM, Wheelock L, Perry D. Pulmonary interstitial glycogenosis. Pediatr Radiol 2010;40(3):361-365 